\title{
Therapeutic efficiency analyses of mandibular advancement devices using polysomnography, smartphone sleep applications, and simple pulse oximetry
}

\author{
(1) Bahadır Ezmek¹, (1) Bülent Pişkin², (1) Cumhur Sipahi ${ }^{1}$ \\ 1 University of Health Sciences Turkey, Gülhane Faculty of Dentistry, Department of Prosthodontics, Ankara, Turkey \\ ${ }^{2}$ Kapadokya University Faculty of Dentistry, Technology of Dental Prosthodontics, Nevsehir, Turkey
}

Date submitted:

28.05.2020

Date accepted:

10.08.2020

Online publication date:

15.03.2021

\section{Corresponding Author:}

Bahadır Ezmek MSc, DDS, PhD, University of Health Sciences

Turkey, Gülhane Faculty of Dentistry, Department of Prosthodontics, Ankara, Turkey

bahadir.ezmek@sbu.edu.tr, bezmek@gmail.com

ORCID:

orcid.org/0000-0002-1651-3260

Keywords: Snoring, obstructive sleep apnea, mandibular advancement device, pulse oximeter device, smartphone sleep applications

\begin{abstract}
Aims: Oral appliances are non-invasive solutions for obstructive sleep apnea (OSA). The aim of the present study was to determine the therapeutic efficiency of mandibular advancement devices (MAD), using polysomnography (PSG), smartphone sleep applications (SSA), and simple pulse oximetry (SPO) for OSA patients.

Methods: Totally 18 mild-moderate OSA patients (8 females and 10 males, aged between 34 and 83 years) with subjective snoring complaints were selected. The diagnostic PSG tests were accepted as initial PSG. Each patient was subjected to SPO and SSA at 3 different nights at home. The mean diagnostic values of oxygen desaturation index (ODI), snoring intensity score (SIS) and snoring percentage (SP) were obtained. Non-titratable-customized MADs (NTC-MAD) with $60-75 \%$ of maximal mandibular protrusion were fabricated. SPO and SSA measurements were repeated at the $1^{\text {st }}, 4^{\text {th }}, 12^{\text {th }}$, and $24^{\text {th }}$ weeks of the treatment process. At the $24^{\text {th }}$ week of the treatment, the PSG tests were repeated and all PSG, SPO, and SSA values were compared with initial diagnostic values.
\end{abstract}

Results: There was no significant difference in body mass index and neck circumference values during the treatment $(p>0.05)$. The success rate of NTC-MAD treatment was about $68.7 \%$ according to the success criterion of MAD treatment as the decrease of apnea-hypopnea index below 5 per hour. ODI, SIS, and SP values showed a significant decrease during the treatment $(p<0.01)$. A maximum decrease in SPO and SSA data were obtained the $12^{\text {th }}$ week of NTCMAD use $(p<0.05)$.

Conclusions: NTC-MADs significantly decreased snoring intensity and frequency and may be accepted as effective devices in the treatment of OSA.

\section{Introduction}

Obstructive sleep apnea (OSA) is one of the common syndromes of sleep-related breathing disorders according to the "International Classification of Sleep Disorders-3" $(1,2)$. OSA, which causes snoring and sleep fragmentations, resulting in excessive day time sleepiness, originates from apneas and hypopneas (2). OSA severity is defined as mild for apneahypopnea index $(\mathrm{AHI}) \geq 5$ and $<15$, moderate for $\mathrm{AHI} \geq 15$ and $\leq 30$, and severe for $\mathrm{AHI}>30$ events/h (2).

The principal treatment methodology for OSA patients is positive airway pressure (PAP) (2,3). In patients with mild to moderate OSA, oral appliances such as mandibular advancement devices (MADs) and tongue advancement devices are alternately indicated especially for patients intolerant to continuous PAP therapy (3).

The American Academy of Dental Sleep Medicine (3) recommended titratable-customized MADs (TC-MAD) for patient comfort and the ability to permit modifications in the amount of mandibular protrusion for treatment efficacy. However, several studies (4-7) demonstrated similar therapeutic efficiency of nontitratable-customized MADs (NTC-MADs) in the treatment of simple snoring (SS) and OSA.

The treatment efficacy of oral appliances is generally identified by polysomnography (PSG). Important physiological 
sleep markers such as $\mathrm{AHI}$, oxygen desaturation index (ODI), and respiratory disturbance index are obtained with PSG $(2,3,8)$. However, the rareness of qualified PSG centers and late appointment times are still the main factors restricting its usage. Pepin et al. (9) proposed measurement and comparison of snoring sound magnitudes as an alternative method for the follow-up of SS and OSA patients and claimed that at least $15 \%$ of snorers had an $\mathrm{AHI}>15$ events/h. It was shown that the acoustic characteristics of snoring differed between SS and OSA patients (10).

The latest remarkable developments in the telecommunication industry and smartphone technologies have enabled to monitor and compare magnitudes of snoring at home by using a wide range of smartphone sleep applications (SSA). Manufacturers of SSA claim that these applications enable users to record snoring sounds, snoring frequencies, and snoring intensities.

The effect of oral appliances on SS and OSA has been studied extensively by using PSG, type-3 home sleep study device, and subjective questionnaires. However, there are no studies in the literature evaluating the therapeutic effects of MADs in OSA patients using both SSA and simple pulse oximeter (SPO). The aim of the present study was to determine the efficacy of NTC-MAD therapy on snoring intensity, snoring frequency, and oxygen desaturation periods in patients with snoring problems using SSA and SPO methods.

\section{Methods}

The present study was approved by the Clinical Trials Ethics Committee of Gulhane Military Medical Academy (Ankara, Turkey) (2015-KAEK-84/ 24 ${ }^{\text {th }}$ December 2015) and was conducted in accordance with the Declaration of Helsinki. Informed consent was obtained from all individual participants included in the study. The present study was registered at ClinicalTrials.gov (NCT04427111). The participants were selected from OSA patients presenting with subjective snoring $(n=37)$ to the Department of Prosthodontics between December 2015 and January 2017. The exclusion criteria for the study were; having severe OSA, having previous surgery for OSA, and carrying a high risk of cardiovascular, respiratory, neurological or psychiatric disorders $(n=12)$. Patients with an inadequate dental anchor for MAD treatment, temporomandibular joint dysfunction, and Angle Class 3 maxillomandibular relation $(n=7)$ were also excluded. OSA patients who were not eligible for MAD treatment were informed and directed to other treatment options. Totally 18 mild-moderate OSA patients with subjective snoring complaints were included in the study. Of 18 patients, eight patients $(44.4 \%)$ were female and 10 patients were $(56.6 \%)$ male. The average age of the patients was 49.33 years, ranging between 34 and 83 years.

PSG, ODI, and SSA values were used to determine and compare the therapeutic efficacy of NTC-MAD treatment in the present study. The diagnostic PSG values of the subjects (before NTC-MAD use) were accepted as initial PSG (PSG-i) values. Final PSG (PSG-f) measurements were performed after 24 weeks of NTC-MAD treatment with NTC-MADs in situ. Body mass index (BMI) and neck circumference values were also recorded before NTC-MAD treatment and at the $24^{\text {th }}$ week of the treatment of NTC-MAD. The success criterion of NTCMAD treatment was defined as the decrease of AHI below 5 per hour. To detect a reduction in AHI, a minimum sample size of 13 participants was considered to obtain $80 \%$ power at a $95 \%$ confidence to demonstrate a significant difference in $\mathrm{AHI}$ of three months after the NTC-MAD therapy, as previously reported (4). A $10 \%$ dropout rate was assumed. It was indicated that 15 pairs of subjects demonstrated the therapeutic effect of NTC-MAD in the present study.

To determine initial ODI (ODI-i) values, all subjects were first informed about the proper usage of SPO (Beurer PO 80, BEURER GmbH, Ulm, Germany) in detail. Subjects were instructed to press the start button and attach the device's probe on the extremity of their index finger before falling asleep. To determine initial SSA values, all subjects were first informed about the proper usage of SnoreLab SSA software (Reviva Softworks Ltd., London, UK). Then, the subjects were instructed to download SSA on their smartphones. All subjects were recommended to place their smartphones next to their bed with the microphone facing towards the subjects, to keep the charger connected, and to sleep alone at the quietest room of the home during SSA recordings. Also, the subjects were instructed to use SSA and SPO in-home environment during sleep for three nights due to minimizing the environmental impact on recordings. The average SSA and SPO values obtained in three nights were calculated and initial mean values of ODI, snoring intensity score (SIS), and snoring percentage (SP) were obtained.

After obtaining ODI-i and SSA values, NTC-MADs were fabricated. Irreversible hydrocolloid impressions (Italgin Chromatic Alginate, BMS, Capponoli, Italy) were made from both dental arches. The impressions were poured with type 3 dental stone (Denstone 3, Ata Yapı Ürünleri San. ve Tic. Ltd., Turkey). Autopolymerizing acrylic resin splints (Self Curing for Ortho Chrystal, BMS, Capponoli, Italy) in $2-2.5 \mathrm{~mm}$ thickness were fabricated on the models of upper and lower jaws and were tried intraorally. Interferences on maximal retrusive and protrusive positions were eliminated. Maximal mandibular protrusion value (MMPV) and retrusive positions were marked on splints (Figure 1A, 1B). To determine efficient mandibular protrusion value (EMPV), MMPV was measured for each patient and the $60-75 \%(4,11)$ of MMPV was calculated and marked (Figure 1C). An interincisal space of $6 \mathrm{~mm}$ was set for each patient (4). The most comfortable protruded mandibular position situated between 60 and $75 \%$ of MMPV was determined according to 
patient response and registered with wax rims. (Figure 1D) The registered models were mounted on a semi-adjustable articulator (AAA43002 CT, Amann Girrbach Artex, Koblach, Austria). The upper and lower acrylic resin splints were secured to each other in the posterior region with auto polymerizing acrylic resin and NTC-MADs were obtained. Subsequently, the NTC-MADs were trimmed, polished, and placed on dental arches (Figure $1 \mathrm{E}, 1 \mathrm{~F})$. The patients were instructed to wear their NTC-MAD every night. After the initial of NTC-MAD therapy, each patient performed the previously described ODI and SSA procedures at the $1^{\text {st }}, 4^{\text {th }}, 12^{\text {th }}$, and $24^{\text {th }}$ weeks of the treatment protocol.

Consequently, totally 17 measurement values for each subject were recorded; PSG-i values, PSG-f values, ODI-i values, ODI $1^{\text {st }}$ week values $(\mathrm{ODI}-1 \mathrm{w}), \mathrm{ODI} 4^{\text {th }}$ week values $(\mathrm{ODI}-4 \mathrm{w}), \mathrm{ODI} 12^{\text {th }}$ week values $(\mathrm{ODI}-12 \mathrm{w})$, ODI $24^{\text {th }}$ week values (ODI-24w), initial SIS (SIS-i) values, SIS $1^{\text {st }}$ week values (SIS-1w), SIS $4^{\text {th }}$ week values (SIS-4w), SIS $12^{\text {th }}$ week values (SIS-12w), SIS $24^{\text {th }}$ week values (SIS-24w), initial SP values $(\mathrm{SP}-\mathrm{i}), \mathrm{SP} 1^{\text {st }}$ week values $(\mathrm{SP}-1 \mathrm{w}), \mathrm{SP} 4^{\text {th }}$ week values $(\mathrm{SP}-$ $4 \mathrm{w})$, SP $12^{\text {th }}$ week values $(\mathrm{SP}-12 \mathrm{~W})$ and SP $24^{\text {th }}$ week values (SP-24w) were obtained.

\section{Statistical Analysis}

All statistical analyses were performed with the SPSS 22.0 software package (SPSS Inc., Chicago, III). Continuous variables were expressed as the means with standard deviations. The

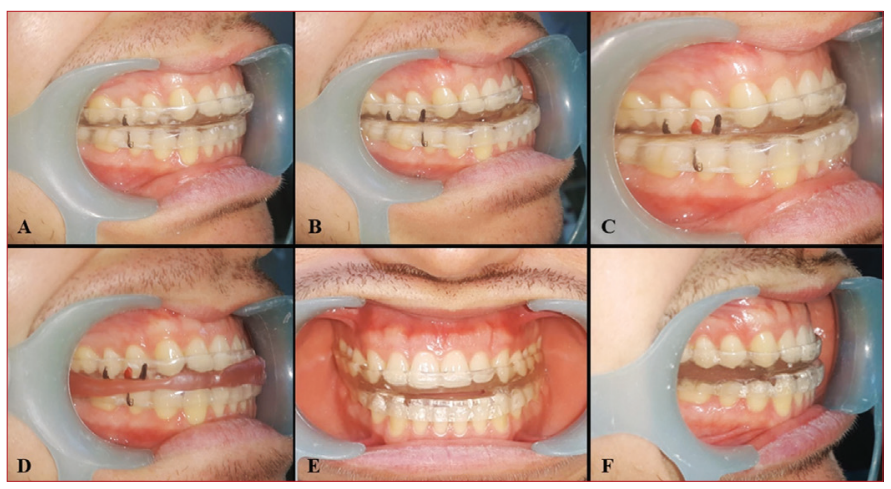

Figure 1. Setting the mandibular protrusion rate at $60-75 \%$ of the maximal mandibular protrusion. A) Retrusive position, B) maximal mandibular protrusion value (MMPV), C) efficient mandibular protrusion value (EMPV) (60-75\% of MMPV). D) Fixing EMPV with wax rims. E and F) Intraoral views of non-titratable customized mandibular advancement device
Kolmogorov-Smirnov test was used to confirm that the data were within the ranges of normal distribution. The t-test (two-tailed for paired samples) was used to compare the differences between initial and final values. To evaluate the effect of MAD on ODI, $\mathrm{SIS}$, and SP, one-way repeated measures analysis of variance test (ANOVA) was used. The Bonferroni-Holm correction was used to correct the multiple comparisons. The type 1 error probability associated with this test was set as $(\alpha=0.05)$.

\section{Results}

All patients completed the study protocol. No significant difference was found between the mean BMI and neck circumference values comparing the $1^{\text {st }}(B M l=29.62 \pm 5.56 \mathrm{~kg} /$ $\mathrm{m}^{2}$ and neck circumference $=38.22 \pm 4.12 \mathrm{~cm}$ ) and $24^{\text {th }}$ weeks $\left(\mathrm{BMI}=28.84 \pm 5.10 \mathrm{~kg} / \mathrm{m}^{2}\right.$ and neck circumference $=38.22 \pm 4.12$ $\mathrm{cm}$ ) of the treatment $[\mathrm{t}(17)=1.748, \mathrm{p}=0.98$ and $\mathrm{t}(17)=1.758$, $\mathrm{p}=0.97$ respectively].

PSG-i and $24^{\text {th }}$ week (PSG-f) mean PSG values were shown in Table 1. In the comparison of PSG-i and PSG-f values, it was determined that all $\mathrm{AHI}, \mathrm{ODI}$, and $\mathrm{SI}$ values were significantly decreased. The average decrease of $\mathrm{AHI}, \mathrm{ODI}$, and SI values were $5.67 \pm 3.66 / \mathrm{h}(p<0.001), 4.25 \pm 3.49 / \mathrm{h}(\mathrm{p}<0.001)$ and $51.48 \pm 51.25 / \mathrm{h}(p=0.005)$, respectively. According to PSG results, the success of NTC-MAD treatment was $68.7 \%$ for OSA patients.

The mean ODI, SIS, and SP values were depicted in Table 2. Intragroup comparison of ODI, SIS, and SP at 5 different time intervals (ANOVA) showed a significant difference $(p<0.001)$. A significant difference was found between ODI-i and ODI-1w, ODI-4w, ODI-12w, and ODI-24w $(p<0.05)$. The highest decrease in ODI values was reached at the $12^{\text {th }}$ week of the treatment. No significant difference was found between ODI-12w and ODI$24 \mathrm{w}(\mathrm{p}=0.181)$ (Figure 2A). As with ODI values, a significant difference was also found between SIS-i and SIS-1w, SIS- $4 \mathrm{w}$, SIS-12w, and SIS-24w $(p<0.001)$ except SIS-12w and SIS-24w $(p=0.113)$ (Figure 2B).

Intragroup comparison of SP values showed that there was a significant difference at 5 different time intervals $(p<0.001)$. The Bonferroni-Holm correction displaced no significant difference between SP-i and SP-1w ( $p=0.56), S P-4 w$ and SP-12w $(p=1)$ and SP-12w and SP-24w ( $p=0.510)$ (Figure $2 C)$.

\begin{tabular}{|c|c|c|c|c|}
\hline & PSG-i & PSG-f & t value & $p$ value \\
\hline $\mathrm{AHI}$ (events/h) & $10.59 \pm 5.23$ & $4.91 \pm 2.6$ & 6.574 & $<0.001^{*}$ \\
\hline SI (events/h) & $173.17 \pm 131.93$ & $122,26 \pm 31,18$ & 3.48 & $0.005^{\star}$ \\
\hline
\end{tabular}




\section{Discussion}

The objective of the present study was to determine the efficacy of NTC-MAD therapy on snoring intensity, frequency, and oxygen desaturation periods in mild-moderate OSA patients with snoring problems. For the differential diagnosis of patients referring to snoring complaints, $\mathrm{AHI}$ was accepted to be the main physiological sleep parameter in the present study. Besides, the $\mathrm{AHI}$ values were also used for the evaluation of the NTC-MAD's treatment success in OSA patients $(2,3)$.

MAD treatment success rate determined by $\mathrm{AHI}$ varies between 19\% (12) and 71\% (4). Age, gender, BMI, the severity level of OSA, and MAD type may influence the success of MAD

\begin{tabular}{|c|c|c|c|c|}
\hline & $\begin{array}{l}\text { Time } \\
\text { interval }\end{array}$ & Mean $\pm S D$ & f value & $p$ value \\
\hline \multirow{5}{*}{ ODI } & ODI-i & $7.82 \pm 5.39$ & \multirow{5}{*}{25.17} & \multirow{5}{*}{$p<0.001^{*}$} \\
\hline & ODI-1w & $6.62 \pm 4.48$ & & \\
\hline & ODI-4w & $4.37 \pm 2.99$ & & \\
\hline & ODI-12w & $3.5 \pm 2.67$ & & \\
\hline & ODI-24w & $3.14 \pm 2.32$ & & \\
\hline \multirow{5}{*}{ SIS } & SIS-i & $72.96 \pm 31.14$ & \multirow{5}{*}{39.45} & \multirow{5}{*}{$p<0.001^{*}$} \\
\hline & SIS-1w & $61.09 \pm 26.97$ & & \\
\hline & SIS-4w & $48.85 \pm 24.35$ & & \\
\hline & SIS-12w & $40.20 \pm 22.33$ & & \\
\hline & SIS-24w & $36.03 \pm 22.14$ & & \\
\hline \multirow{5}{*}{ SP } & $S P-i$ & $32.61 \pm 17.29$ & \multirow{5}{*}{21.46} & \multirow{5}{*}{$p<0.001^{*}$} \\
\hline & SP-1w & $29.40 \pm 14.60$ & & \\
\hline & SP-4w & $21.29 \pm 11.95$ & & \\
\hline & SP-12w & $19.81 \pm 10.64$ & & \\
\hline & SP-24w & $17.35 \pm 8.97$ & & \\
\hline $\begin{array}{l}\text { Indice } \\
\text { SD: S } \\
\text { intens }\end{array}$ & $\begin{array}{l}\text { significant } \\
\text { dard deviatic } \\
\text { score, SP: S }\end{array}$ & $\begin{array}{l}\text { nges for intragrou } \\
\text { ODI: Oxygen des } \\
\text { ing percentage }\end{array}$ & $\begin{array}{l}\text { mparison } \\
\text { tion index, }\end{array}$ & $\begin{array}{l}\text { NOVA). } \\
\text { IS: Snoring }\end{array}$ \\
\hline
\end{tabular}

treatment $(3,13,14)$. It is emphasized that EMPV is the most determinative parameter affecting customized MAD treatment success (3). Aarab et al. (4) reported that $50 \%$ to $75 \%$ of MMPV had higher treatment efficiencies. Marklund et al. (11) reported that using more than $75 \%$ of MMPV increased the complication probability.

In the present study, the NTC-MADs were fabricated by using $60-75 \%$ of MMPV to provide optimal treatment efficacy and to minimize the side effects. All patients use the appliances appropriately without side effects that restrict the use of appliances. The treatment success of the present study was similar to those in which EMPV was expressed as being the 60$75 \%$ of MMPV, either with TC-MADs or NTC-MADs $(4,6,15-17)$. In these studies, the success of the MAD treatment is determined by full night PSG. However, the insufficient number of qualified sleep laboratories and the expensiveness of the PSG procedure obliged the clinicians to use home testing devices for the diagnosis and follow-up of sleep disorders. One of these home testing devices is SPO (18). SPOs are capable of calculating the ODI and monitoring automatically the desaturation fluctuations during sleep. The major concern about ODI is that hypopneas and short apneas do not always cause a 3-4\% decrease in oxygen saturation. In such cases, the ODI parameter is far from being determinative (18).

Due to the coherence of $\mathrm{AHI}$ and $\mathrm{ODI}$ values in morbid obesity, ODI was especially used for the follow-up procedures of OSA in morbidly obese patients with $\mathrm{AHI}>10$ (19). Recently, Ernst et al. (20) reported that the correlation between $\mathrm{AHI}$ and ODI was larger in patients with normal weight and proportionally decreased by the increase in BMI. This result highlighted the important role of ODI during the follow-up of OSA patients.

In the present study, a significant decrease in ODI (4.68 events/h) was found using NTC-MADs. Incoherence with our study, Ghazal et al. (21) reported a decrease of 4.50 events/h in ODI with TC-MADs. Similarly, Barnes et al. (22) found a decrease of 4.30 events/h in ODI with TC-MADs. Zhou and Liu
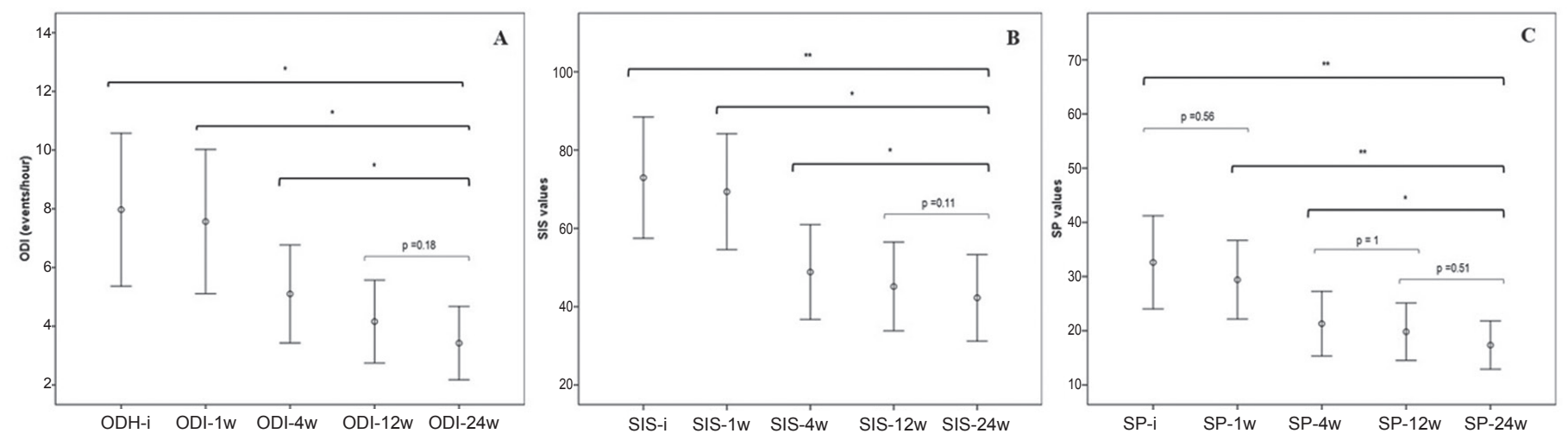

Figure 2. Multiple comparisons of simple pulse oximeter and smartphone sleep application values (Bonferroni-Holm correction) $\left({ }^{*} p<0.05,{ }^{* *} p<0.01,1\right.$. standard deviation). A) Oxygen desaturation index, B) snoring intensity score, C) snoring percentage ODI: Oxygen desaturation index, SIS: Snoring intensity score, SP: Snoring percentage 
(6) reported that compared to TC-MADs, NTC-MADs achieved an equivalent reduction in ODI in adult patients with OSA and reported a decrease of 25.00 events per hour in ODI with the use of an NTC-MADs. The authors physiologically determined individual EMPVs using the PSG test and titration for each patient. That proper method for determining EMPV explains the huge difference in ODI values compared to the present study.

In all of the above-mentioned studies, the mean differences in ODI were only determined at the end of the $12^{\text {th }}$ or $24^{\text {th }}$ weeks. There is not any study that reported the mean ODI difference in periodical time intervals within the treatment procedure. According to the results of the present study, it can be deduced that the maximum reduction in ODI can be achieved in 12 weeks.

MADs are not only indicated for the treatment of OSA patients but also for SS patients. The effects of MADs on snoring were mostly assessed by subjective tests such as questionnaires and visional analog scale $(3,5)$. However, some researchers suggested using sensors and microphones in a soundproofing environment for recording and evaluating snoring sounds objectively $(10,23)$. Snoring sound frequency could be evaluated as an important parameter for differential diagnosis between SS and OSA because the snoring sounds of OSA patients recorded in sleep laboratories have a frequency higher than $800 \mathrm{~Hz}(10)$ and SS patients usually have frequencies between 110 and 190 $\mathrm{Hz}$ (below $500 \mathrm{~Hz}$ ) (23).

Remarkable technological advancement of communication provides recording snoring sounds at the patient's home by using smartphones. And also, SSAs monitor the snoring sounds as numeric data. Although there are many SSAs, few studies report about the reliability of SSAs. An excellent positive correlation was reported between SSAs and sleep laboratory sound recordings of $93 \%$ to $96 \%$ in a soundproof environment $(24,25)$.

According to studies evaluating snoring sounds, the MAD treatment decreased SIS $(26,27)$. Walker-Engström et al. (26) determined that NTC-MADs with $75 \%$ of MMPV caused a higher decrease in SIS. Besides, Bloch et al. (27) stated that NTC-MADs provided a greater reduction in subjective snoring intensity than TC-MADs. In the present study, SIS values started to decrease just from the $1^{\text {st }}$ week of NTC-MAD treatment. O'Sullivan et al. (28) reported that MADs reduced SIS even at the first night. Smith and Battagel (29) reported that the use of MAD for a month caused a $64 \%$ reduction in SIS. However, the SIS reduction rate $(43 \%)$ in this study was not as high as theirs. That difference may be explained with the determination difference of EMPV existing between two studies. While Smith and Battagel (29) used one-night titration to determine EMPV, a standard EMPV (60-75\% of MMPV) was used in the present study. Considering the obtained SSA data, it was determined that the maximum reduction in SIS with the use of NTC-MADs could be obtained at the $12^{\text {th }}$ week.
It was determined in the present study that SP significantly decreased $(39 \%)$ at the $4^{\text {th }}$ week and that level remained constant till the end of the study. Umemoto et al. (7) and Lee et al. (30) reported a $40 \%$ and $34 \%$ decrease in SP values, respectively, with the use of NTC-MADs. However, Umemoto et al. (7) found a decrease of $9 \%$ in SP values with TC-MADs while that of NTC-MADs was $40 \%$. The authors concluded that due to their more stable and compact structure that keeps the mandible at a constant protrusive position, NTC-MADs were much more efficient in SP than TC-MADs.

Numerous sleep medicine authors recommended the use of TC-MADs due to some advantages like providing the titration of MADs to optimize its efficiency, keeping the mandible more flexible, and causing fewer side effects $(2,3)$. However, NTCMADs were used in the present study as in some previous studies $(3,5,26,27)$ reporting that the treatment efficiencies of NTC-MADs and TC-MADs were similar. Further, some studies declared that NTC-MADs were more efficient in snoring due to structural characteristics $(6,7)$. Besides, NTC-MADs are much more economic devices compared to TC-MADs, thus, lowcosting NTC-MADs were used for SS and OSA patients with snoring complaints. The other limitations are that no information exists about how to calibrate SnoreLab to calculate SIS and SP and that SSAs may be affected by ambient sounds (25). A wide range of smartphones with various technological properties may lead to differences in the determination of MAD treatment success. Also, the age and susceptibility of the patients to the technology may affect the results of the sleep studies.

SSAs are still very new and there is not enough reliable information about them. However, technological developments can be considered as a messenger soon that SSAs, which have advanced algorithms that are less affected by smartphone hardware and ambient sounds, will take their place in the markets.

\section{Conclusion}

Within the limitations of this in vivo study, the following conclusions were drawn:

1. The use of NTC-MADs for 24 weeks was found to be an efficient method for decreasing AHI, ODI, SI, SIS, and SP.

2. Significant reduction in ODI and SIS was observed at the end of the $1^{\text {st }}$ week and continued to decrease regularly until the $12^{\text {th }}$ week.

3. A significant decrease in SP was determined at the $4^{\text {th }}$ week.

It was concluded that NTC-MADs significantly decreased snoring intensity and frequency and they are effective devices in the treatment of SS and OSA. 


\section{Ethics}

Ethics Committee Approval: The trial was approved by Clinical Trials Ethics Committee of Gülhane Military Medical Academy in Ankara, Turkey (protocol number: 2015-KAEK-84, date: $24^{\text {th }}$ December 2015).

Informed Consent: All patients gave their written informed consent to participate in the study.

Peer-review: Externally and internally peer-reviewed.

\section{Authorship Contributions}

Surgical and Medical Practices: B.E., Concept: B.E., B.P., C.S., Design: B.E., B.P., C.S., Data Collection or Processing: B.E., B.P., Analysis or Interpretation: B.E., Literature Search: B.E., Writing: B.E., B.P., C.S.

Conflict of Interest: No conflict of interest was declared by the authors.

Financial Disclosure: The authors declared that this study received no financial support.

\section{References}

1. Sateia MJ. International classification of sleep disordersthird edition highlights and modifications. Chest. 2014;146:1387-1394.

2. Epstein LJ, Kristo D, Strollo PJ, et al. Clinical guideline for the evaluation, management and longtime care of obstructive sleep apnea in adults. J Sleep Med. 2009;5:263-276.

3. Ramar K, Dort LC, Katz SG, et al. Clinical practice guideline for the treatment of obstructive sleep apnea and snoring with oral appliance therapy: an update for 2015. J Clin Sleep Med. 2015;11:773-827.

4. Aarab G, Lobbezoo F, Hamburger HL, Naeije M. Effects of an oral appliance with different mandibular protrusion positions at a constant vertical dimension on obstructive sleep apnea. Clin Oral Investig. 2010;14:339-345.

5. Blanco J, Zamarron C, Abeleira Pazos MT, Lamela C, Suarez Quintanilla D. Prospective evaluation of an oral appliance in the treatment of obstructive sleep apnea syndrome. Sleep Breath. 2005;9:20-25.

6. Zhou J, Liu YH. A randomized titrated crossover study comparing two oral appliances in the treatment for mild to moderate obstructive sleep apnoea/hypopnoea syndrome. J Oral Rehabil. 2012;39:914-922.

7. Umemoto $\mathrm{G}$, Toyoshima $\mathrm{H}$, Yamaguchi $\mathrm{Y}$, Aoyagi $\mathrm{N}$, Yoshimura C, Funakoshi K. Therapeutic Efficacy of TwinBlock and Fixed Oral Appliances in Patients with Obstructive Sleep Apnea Syndrome. J Prosthodont. 2019;28:830-836.

8. Berry RB, Budhiraja R, Gottlieb DJ, et al. Rules for scoring respiratory events in sleep: update of the 2007 AASM Manual for the Scoring of Sleep and Associated Events. Deliberations of the Sleep Apnea DefinitionsTask Force of the American Academy of Sleep Medicine. J Clin Sleep Med. 2012;8:597-619.

9. Pepin JL, Guillot M, Tamisier R, Levy P. The upper airway resistance syndrome. Respiration. 2012;83:559-566.

10. Fiz JA, Jané R, Solà-Soler J, Abad J, García MA, Morera J. Continuous analysis and monitoring of snores and their relationship to the apnea-hypopnea index. Laryngoscope. 2010;120:854-862.

11. Marklund M, Verbraecken J, Randerath W. Non-CPAP therapies in obstructive sleep apnoea: mandibular advancement device therapy. Eur Respir J. 2012;39:12411247.

12. Engleman HM, McDonald JP, Graham D, et al. Randomized crossover trial of two treatments for sleep apnea/hypopnea syndrome: continuous positive airway pressure and mandibular repositioning splint. Am J Respir Crit Care Med. 2002; 166:855-859.

13. Petri N, Svanholt P, Solow B, Wildschiødtz G, Winkel P. Mandibular advancement appliance for obstructive sleep apnoea: results of a randomized placebo controlled trial using parallel group design. J Sleep Res. 2008;17:221 229.

14. Marklund M, Franklin KA. Treatment of elderly patients with snoring and obstructive sleep apnea using a mandibular advancement device. Sleep Breath. 2014;19:403-405.

15. Nerfeldt P, Friberg D. Effectiveness of oral appliances in obstructive sleep apnea with respiratory arousals. J Clin Sleep Med. 2016;12:1159-1165.

16. Anitua E, Durán-Cantolla J, Almeida GZ, Alkhraisat $\mathrm{MH}$. Minimizing the mandibular advancement in an oral appliance for the treatment of obstructive sleep apnea. Sleep Med. 2017;34:226-231.

17. Isacsson G, Fodor C, Sturebrand M. Obstructive sleep apnea treated with custom-made bibloc and monobloc oral appliances: a retrospective comparative study. Sleep Breath. 2017;21:93-100.

18. Jobin V, Mayer P, Bellemare F. Predictive value of automated oxygen saturation analysis for the diagnosis and treatment of obstructive sleep apnoea in a homebased setting. Thorax. 2007;62:379-380.

19. Malbois M, Giusti V, Suter M, Pellaton C, Vodoz JF, Heinzer R. Oximetry alone versus portable polygraphy for sleep apnea screening before bariatric surgery. Obes Surg. 2010;20:326-331.

20. Ernst G, Bosio M, Salvado A, Dibur E, Nigro C, Borsini E. Difference between apnea-hypopnea index (AHI) and oxygen desaturation index (ODI): proportional increase associated with degree of obesity. Sleep Breath. 2016;20:1175-1183.

21. Ghazal A, Sorichter S, Jonas I, Rose EC. A randomized prospective long term study of two oral appliances for sleep apnoea treatment. J Sleep Res. 2009;18:321-328.

22. Barnes M, McEvoy RD, Banks S, et al. Efficacy of positive airway pressure and oral appliance in mild to moderate 
obstructive sleep apnea. Am J Respir Crit Care Med. 2004;170:656-664.

23. Cathcart RA, Hamilton DW, Drinnan MJ, Gibson GJ, Wilson JA. Night-to-night variation in snoring sound severity: one night studies are not reliable. Clin Otolaryngol. 2010;35:198203.

24. Camacho M, Robertson M, Abdullatif J, et al. Smartphone apps for snoring. J Laryngol Otol. 2015;129:974-979.

25. Stipping A, Hübers $U$, Emerich M. Apps in sleep medicine. Sleep Breath. 2015;19:411-417.

26. Walker-Engström ML, Ringqvist I, Vestling O, Wilhelmsson $B$, Tegelberg A. A prospective randomized study comparing two different degrees of mandibular advancement with a dental appliance in treatment of severe obstructive sleep apnea. Sleep Breath. 2003;7:119-130.
27. Bloch KE, Iseli A, Zhang JN, et al. A randomized, controlled crossover trial of two oral appliances for sleep apnea treatment. Am J Respir Crit Care Med. 2000;162:246-251.

28. O'Sullivan RA, Hillman DR, Mateljan R, Pantin C, Finucane KE. Mandibular advancement splint: an appliance to treat snoring and obstructive sleep apnea. Am J Respir Crit Care Med. 1995;151:194-198.

29. Smith AM, Battagel JM. Non-apneic snoring and the orthodontist: the effectiveness of mandibular advancement splints. J Orthod. 2004;31:115-123.

30. Lee $\mathrm{CH}, \mathrm{MoJH}, \mathrm{Choi}$ IK, et al. The Mandibular Advancement Device and Patient Selection in the Treatment of Obstructive Sleep Apnea. Arch Otolaryngol Head Neck Surg. 2009;135:439-444. 